\title{
Adsorbed oxygen-induced cluster reconstruction on core-shell Ni@Pt and Pt clusters
}

\author{
Ferensa Oemry ${ }^{\mathrm{a}}$, Hiroshi Nakanishi ${ }^{\mathrm{a}}$, Hideaki Kasai ${ }^{\mathrm{a},{ }^{*},}$, Hiroyoshi Maekawa ${ }^{\mathrm{b}}$, Kazuo \\ Osumi $^{\mathrm{b}}$, Kaoru Sato ${ }^{\mathrm{b}}$ \\ ${ }^{a}$ Department of Applied Physics, Graduate School of Engineering, Osaka \\ University, Suita, Osaka 565-0871, Japan \\ ${ }^{\mathrm{b}}$ Isuzu Advanced Engineering Center, Ltd., Fujisawa, Kanagawa 252-0881, Japan
}

\begin{abstract}
Density-functional calculations were performed to study Pt-O bonding interaction on core-shell Ni@Pt and pure Pt clusters composed of 55 atoms each. Based on oxygen adsorption energy and $v(\mathrm{Pt}-\mathrm{O})$ stretching frequency, the global trend of Pt-O bonding strength in the clusters accommodates the successive order of bridge (and/or hcp-like) $>$ fcc-like $>$ vertex site. Further analysis on oxygen-induced reconstruction effect using first nearest neighbor $(1 \mathrm{NN})$ analysis shows that $1 \mathrm{NN}$ Pt-Pt distribution pattern of surface atoms changes abruptly after oxygen adsorbed. The profiles of distorted 1NN distribution pattern are greatly determined by the position and the number of adsorbed oxygen on the clusters including whether the cluster type is core-shell or not. The broadening distribution pattern clearly indicates deviation of Pt-Pt bond length due to Pt-O bonds formation and the general trend is similar with the one observed in platinum-related spectroscopy studies.
\end{abstract}

Keywords: core-shell Ni@Pt; platinum cluster; oxygen adsorption; reconstruction; first nearest neighbor; DFT

\section{Introduction}

Platinum (Pt) has been playing a major role in diesel oxidation catalyst (DOC) consisting of platinum coated on $\mathrm{Al}_{2} \mathrm{O}_{3}$ support [1]. Due to its high cost and scarce resource, reducing the size of platinum into several nanometers has been considered to be a viable strategy in fabricating low-cost $\mathrm{Pt} / \mathrm{Al}_{2} \mathrm{O}_{3}$ catalysts. However, the method still has several drawbacks because of the facts those smaller Pt nanoparticles are

${ }^{*}$ Corresponding author. Tel : +81-6-6879-7857

E-mail address : kasai@dyn.ap.eng.osaka-u.ac.jp (H. Kasai) 
notoriously susceptible to oxygen poisoning [2], deformation [3], and sintering processes [4,5]. In recent years, several experimental studies have succeeded in synthesizing $\mathrm{NiPt} / \mathrm{A}_{2} \mathrm{O}_{3}$ catalyst that demonstrates better catalytic reactivity in dry reforming of methane (DRM) [6,7], ethanol steam reforming [8,9], and hydrogenation of maleic anhydride (MA) [10] processes. This new type of catalyst is deemed to have a greater chance to improve and enhance the current catalytic oxidation of DOC. However, to the best of our knowledge, the catalyst has never been applied in DOC yet. One of the main hindrance in implementing the catalyst in DOC might stem from the uncertainties on how the Langmuir-Hinshelwood [11] and Eley-Rideal [12] mechanisms, which govern the oxidation processes in DOC, might proceed in the Ni-Pt catalyst. Another critical consideration is the appropriate mixing pattern for the alloyed Ni-Pt metals. It has been well-known that nickel exhibits stronger affinity towards oxygen and surface with high-coverage of $\mathrm{Ni}$ atoms could form robust $\mathrm{Ni}-\mathrm{O}$ bonds stronger than do Pt-O bonds [13], hence, it would further accelerate the deactivation of the catalyst. Therefore, the design of new alloyed Ni-Pt catalyst is required to possess a geometric structure where its outer-most surface is highly dominated by $\mathrm{Pt}$ atoms rather than $\mathrm{Ni}$ atoms.

Core-shell Ni@Pt nanoparticle [14-16] is considered as a suitable solution that can satisfy the requirements above for three reasons: (1) The oxidation reactions would still be carried out on Pt-enriched surface, thus, it imposes no significant change to the general reactions mechanism that occurs in conventional DOC; (2) With a proper $\mathrm{Ni}: \mathrm{Pt}$ stoichiometric ratio, nickel atoms could be covered almost perfectly by $\mathrm{Pt}$ atoms and refrained from making direct contact with oxygen; (3) Another potent advantage is as the size of the nanoparticles increases the shell-to-core atomic ratio decreases, consequently, less Pt loading would be needed in core-shell nanoparticles and reduction in Pt content is feasibly possible [14]. All of those benefits may give a solid justification to endorse core-shell $\mathrm{Ni} @ \mathrm{Pt}$ nanoparticles as prospective catalyst candidate in DOC which is the aim of this present theoretical study.

Recent experimental study on $\mathrm{Pt} / \mathrm{Al}_{2} \mathrm{O}_{3}$ [17] shows that $\mathrm{NO}$ oxidation on the smallest Pt nanoparticle size $(\approx 1 \mathrm{~nm})$ was found to exhibit the lowest NO conversion and concomitantly the most sluggish turn over frequency (TOF). Early experimental work [18] has interpreted this sluggish TOF during NO oxidation due to stronger Pt-O bonds on smaller Pt nanoparticles as further confirmed by DFT study [19]. In accordance to the objective of this study, it would be interesting and important to verify whether the replacement of 1 nm Pt nanoparticles with core-shell Ni@Pt nanoparticles of similar size would be able to significantly reduce the bonding strength of Pt-O bonds or not. Moreover, it is also interesting to clarify the effectiveness of compressive strain 
effect [20] on Pt-Pt bonds in Ni@Pt nanoparticles because there is always a higher possibility that the core-shell nanoparticles might experience a greater surface relaxation during Pt-O bonds formation. Therefore, in this preliminary work, we carry out density functional theory (DFT) calculation to examine Pt-O bonding interaction on $1 \mathrm{~nm}$ core-shell $\mathrm{Ni@Pt} \mathrm{cluster} \mathrm{and} \mathrm{also} \mathrm{the} \mathrm{influence} \mathrm{of} \mathrm{oxygen-induced} \mathrm{cluster}$ reconstruction in reshaping the distribution pattern of first nearest neighbor (1NN) Pt-Pt bond in the cluster. As we will show later the change in 1NN Pt-Pt distribution that caused by adsorbed oxygen is a feature that closely comparable to the depiction of Fourier transformed EXAFS data during platinum oxidation process [21,22]. In order to build some connection between theoretical model of Ni@Pt cluster and the EXAFS measurement above, (non-alloyed) pure Pt cluster of similar size was built as reference for side-by-side comparison with Ni@Pt cluster. Aside from computational cost and limitation considerations, $1 \mathrm{~nm}$ Pt cluster is of particular interest because such cluster has been experimentally synthesized and characterized as shown in many studies $[17,23-25]$.

\section{Computational Methods}

To construct $1 \mathrm{~nm}$ nanoparticles, we adopted the so-called magic cluster (i.e., clusters containing complete shell of atoms) size of 55 atoms [26,27] and found that Mackay icosahedron is the most stable structure among other 55-atom Pt cluster isomers. In designing Ni@Pt cluster, we deliberately evaluated only one stoichiometric ratio of Pt:Ni that proportionally close to 3:1 considering that this ratio can guarantee all surface atoms are composed of $\mathrm{Pt}$ atoms while experimentally it has been demonstrated as the optimum ratio to achieve high catalytic reactivity in oxygen reduction reaction (ORR) [28]. Based on seminal DFT study that carried out by Wang et al [27], it is confirmed that the alloyed 55-atom Ni-Pt cluster is energetically more favorable to accommodate Pt-enriched surface. Thus, this allows us to construct Ni@Pt cluster where 42 out of 55 atoms are positioned on the cluster surface.

All the calculations were performed using VASP program [29-31] by implementing the generalized gradient approximation (GGA) with Perdew-Burke-Ernzerhof (PBE) [32] for the exchange correlation functional and the projector augmented-wave (PAW) method by using a plane-wave cutoff energy of $400 \mathrm{eV}$. Using one $\Gamma$ k-point only, the energies were converged to $1 \mathrm{meV} /$ atom and the ionic relaxation was allowed until the absolute force on each atom was below $0.02 \mathrm{eV} / \AA$. The clusters were simulated in a periodic cubic cell with a size of $25 \AA$ sides, ensuring a sufficient vacuum region to 
separate clusters in neighboring cells. All atoms, except one core atom in the center, were allowed to undergo full relaxation. In order to investigate oxygen-induced reconstruction effect, we have applied two different relaxation schemes for oxygen adsorption calculations, they are: (1) oxygen atom was relaxed and all atoms in the clusters were fixed; (2) oxygen and all atoms in the clusters, except one fixed atom, were fully relaxed.

The oxygen adsorption energy $\left(\mathrm{E}_{\mathrm{ad}}\right)$ is calculated from the total energy difference between Pt cluster with oxygen $\left(\mathrm{E}_{\mathrm{Pt}-\mathrm{O}}\right)$, pure Pt cluster $\left(\mathrm{E}_{\mathrm{Pt}}\right)$, and isolated oxygen $\left(\mathrm{E}_{\mathrm{O}}\right)$

relative to $\frac{1}{2} \mathrm{O}_{2}$ in gas phase $\left(\mathrm{E}_{\mathrm{O}}\right)$ as given by the equation, $\mathrm{E}_{\mathrm{ad}}=\mathrm{E}_{\mathrm{Pt}-\mathrm{O}}-\left(\mathrm{E}_{\mathrm{Pt}}+\mathrm{E}_{\mathrm{O}}\right)($ For core-shell case, $\mathrm{E}_{\mathrm{Pt}-\mathrm{O}}$ and $\mathrm{E}_{\mathrm{Pt}}$ are replaced by $\mathrm{E}_{\mathrm{Ni} / \mathrm{Pt}-\mathrm{O}}$ and $\mathrm{E}_{\mathrm{Ni} / \mathrm{Pt}}$, respectively). To calculate the oxygen vibrational modes, the oxygen atom was displaced from its equilibrium position where its displacement is regulated in accordance with density-functional perturbation theory (DPFT) method [33] that executed within VASP program. In this calculation, all metal atoms which built the clusters remain fixed because those atoms ( $\mathrm{Ni}$ and $\mathrm{Pt}$ ) have weight mass several times heavier than oxygen, therefore they would not be displaced as the oxygen atom vibrates. This approximation would only give a slight deviation to the oxygen vibrational frequencies.

\section{Results and Discussions}

\subsection{Cluster geometric structure and oxygen adsorption energy}

Figure 1 shows the optimized core-shell Ni@Pt (a) and pure Pt (b) clusters where the surface (shell) atoms arrangement gives 20 identical equilateral triangular faces and all of them share similar high-index (111) facet surface. With respect to the stable configuration of 13-atom Ni and Pt clusters [34,35] (see Fig. 1(c)), the inner Ni-Ni bonds $\left(\mathrm{a}_{0}, \mathrm{a}_{1}\right)$ of $\mathrm{Ni} @ \mathrm{Pt}$ cluster are stretched but the inner Pt-Pt bonds $\left(\mathrm{a}_{0}, \mathrm{a}_{1}\right)$ of pure Pt cluster remains unchanged (see Table 1). Interestingly, the average shell Pt-Pt bond distances $\left(\mathrm{a}_{4}\right)$ of core-shell Ni@Pt $(2.68 \AA)$ and pure Pt $(2.79 \AA)$ clusters are closely match the values that obtained from X-ray diffraction (XRD) data, $2.70 \AA$ and $2.77 \AA$, respectively [36,37]. However, the actual shell Pt-Pt bond distances in 55-atom cluster are not homogeneous, most probably, because there are two distinguishable shell $\mathrm{Pt}$ atoms which possess two different nearest neighbor atoms. As seen in Figs. 1(a) and 1(b), those atoms are labeled by A and B letters which stand for atoms with low and high coordination numbers, respectively. Furthermore, the average bond distances for 
A-B and B-B bonds in Ni@Pt case are $2.63 \AA$ and $2.73 \AA$ consecutively, while the obtained values in pure Pt cluster are $2.72 \AA$ and $2.86 \AA$ following the same sequence. It is obvious that all shell Pt-Pt bonds in $\mathrm{Ni} @ \mathrm{Pt}$ cluster are contracted due to the presence of $\mathrm{Ni}$ core atoms. In addition, the values of $\mathrm{a}_{4}$ which are provided in Table 1 are gained by taking the average value of A-B and B-B bond lengths.

Because the clusters have high geometric symmetry, investigation to locate the preferable adsorption sites for oxygen atom can be concentrated on one equilateral triangular face where 6 adsorption sites are considered as indicated in Fig. 2 for each corresponding cluster. The 6 adsorption sites have taken into account all possibilities of Pt-O bond configurations that may exist. The oxygen adsorption energies are pictorially summarized in Fig. 3 for each assigned site. For all sites, oxygen adsorption energies in pure Pt cluster are lower than those energies in $\mathrm{Ni@Pt} \mathrm{for} \mathrm{either} \mathrm{reconstructed} \mathrm{or} \mathrm{rigid}$ cluster case. Furthermore, the oxygen adsorption energies in the reconstructed pure $\mathrm{Pt}$ cluster, which are spanning between $-3.45 \mathrm{eV}$ to $-4.60 \mathrm{eV}$, are quite reasonable in comparison with thermal desorption spectroscopy (TDS) work on $\operatorname{Pt}(111)$ surface [38]. In that study, the heat of desorption at oxygen coverage ranging from 0.2 of saturated coverage (upper limit) into nearly zero (lower limit) is within the limit of 250-500 $\mathrm{kJ} / \mathrm{mol}$ or equivalent to $2.59-5.18 \mathrm{eV}$. Further analysis provides that while majority of the adsorption sites give oxygen adsorption energies closer to or lower than $-4 \mathrm{eV}$, oxygen atom that adsorbed on vertex B site has the weakest adsorption energy. In addition, the site number 5 (bridge $\mathrm{B}$ ) is not favorable for Pt-O bonds formation and oxygen on those sites are more likely to drift away to hcp-like sites during structural optimization. The reason could be the activation energy barriers of the reaction pathway between bridge B and hcp-like sites are minuscule, as consequence, the metastable adsorbed oxygen atom on bridge B sites could easily slide off to hcp-like sites.

\subsection{Adsorption site preference and stretching frequency}

In stepped Pt(111) surface, the dissociated oxygen atoms are widely known to be attracted subsequently to edge-bridge $>$ fcc $>$ hcp $>$ bridge $>$ atop site [38-41]. Similar observation is also found in $\operatorname{Pt}(335)$ and $\operatorname{Pt}(321)$ surfaces. However, for 55-atom cluster case, as the definite boundary line that divides edge and three-fold hollow sites region becomes blurred, the oxygen adsorption energy gap between those sites is also getting smaller (see Fig. 3). Accordingly, the given adsorption energies are less reliable to draw the sequence of most-to-least-stable adsorption sites for oxygen atom in both $\mathrm{Ni@Pt}$ and pure Pt clusters. Recently, potential energy surface (PES) that based on 
computational work on $\operatorname{Pt}(321)$ [42] has successfully elaborated the oxygen adsorption sites preference that previously proposed by HREELS and TDS measurements [43] where the results of both experimental and theoretical works lead to similar conclusions. For this reason, $v(\mathrm{Pt}-\mathrm{O})$ stretching frequencies for 5 adsorption sites on the clusters were calculated and listed in Table 2.

It is clearly noticeable that the $v(\mathrm{Pt}-\mathrm{O})$ stretching frequency values in $\mathrm{Ni@Pt} \mathrm{cluster}$ are generally higher than their counterparts in pure Pt cluster for all sites. The discrepancy is mainly caused by the influence of $\mathrm{Ni}$ atoms as previously explained by Jacob et al. [44]. Apart from that, our work and theirs also share one common similarity which agrees that higher vibrational frequencies do not always proportionally correlated to higher oxygen adsorption energy or vice versa. Through careful analysis, there is a clear decreasing trend of frequency gap between edge-bridge and three-fold hollow sites as this tendency systematically obeys the successive order of $\mathrm{Pt}(335)>$ pure $\mathrm{Pt}>\mathrm{Ni} @ \mathrm{Pt}$ as shown in Table 2 (the right-most column). This phenomenon of narrowing frequency gap can be interpreted as an indication that the edge-bridge and three-fold hollow sites in the clusters are starting to impose similar Pt-O bonding strength due to geometry and alloying effects.

The oxygen on vertex sites is particularly interesting because it gains the highest $v(\mathrm{Pt}-\mathrm{O})$ stretching frequencies above $700 \mathrm{~cm}^{-1}$ as compared with other sites. Previously, oxygen species with vibrational frequency values around $700 \mathrm{~cm}^{-1}$ had been observed by McCellan et al [43] and Wang et al [45] on $\mathrm{Pt}(321)$ and $\mathrm{Pt}(335)$ surfaces, respectively. Both authors concurred that the values emerge from metastable oxygen species at step sites. However, there was a disagreement between them whether the oxygen species that produce values near $700 \mathrm{~cm}^{-1}$ are in atomic or molecular state. Judging from their description (particularly Figure 7 in the work of Wang et al), we argue that the metastable oxygen species might have located at transition state (TS) because oxygen species at that state could undergo recombination to revert back to its molecular state or completely transforms itself into independent oxygen atoms. To prove our preconception, we have provided $\mathrm{O}-\mathrm{O}$ stretching frequency values at TS in small $\mathrm{Pt}_{4}$ and $\mathrm{Pt}_{10}$ clusters [46] as additional data for comparative analysis. The O-O bond distances in $\mathrm{Pt}_{4}$ and $\mathrm{Pt}_{10}$ clusters at TS are subsequently $1.78 \AA$ and $2.2 \AA$. These bond lengths dismiss the conjecture that refers the $700 \mathrm{~cm}^{-1}$ to the $\mathrm{O}-\mathrm{O}$ stretching frequency at peroxo state since the $\mathrm{O}-\mathrm{O}$ bond length at that state is around 1.42-1.43 $\AA$ [47]. In corresponding to oxygen atom on vertex $\mathrm{A}$ and vertex $\mathrm{B}$ sites, the situation can be interpreted as the oxygen atom is at metastable state which could later relocate onto more preferable sites such as bridge or hcp-like site if the conditions are permitted. 
Moreover, based on the data that presented in Table 3, the adsorption sites that could deliver Pt-O bond distances close to the experimental value [22] are only bridge, hcp-like, and fcc-like sites. This proves that adsorbed oxygen atom in real experimental conditions is less likely to occupy vertex sites for long periods of time. In summary, the global trend of Pt-O bonding strength in the clusters accommodates the following successive order of bridge (or hcp-like) $>$ fcc-like $>$ vertex A $>$ vertex B.

\subsection{Cluster reconstruction in one-atom oxygen case}

G.A. Somorjai and coworkers [48-50] reported that surface reconstruction could occur during chemical processes. In those studies, they proposed that surface metals are not completely rigid but instead have certain levels of flexibility and can undergo surface deformation where the surface of nano-structured cluster is considered to be the most flexible one. $\mathrm{Xu}$ et al [51] later confirmed the prediction of experimental studies above by showing that small Pt clusters could undergo major structural change at high oxygen coverage. In relation to our work, we studied cluster reconstruction that deviates Pt-Pt bond lengths in core-shell Ni@Pt and pure Pt clusters gives similar indicative trends that can be unambiguously compared to the reported works of spectroscopy measurements.

With the advent of new technical methods in XRD and XAFS experiments $[21,22,52]$, it is now becomes possible to monitor real-time surface reconstruction on $\mathrm{Pt}$ nanoparticles when they are interacting with oxygen. In those experimental studies, Allen et al [21] and Imai et al [22] demonstrate how the amplitude of first nearest neighbor (1NN) Pt-Pt bond distribution that plotted in Fourier transform (FT) graph of EXAFS is decreasing while at the same time another amplitude that refers to Pt-O bonds is gradually increasing as the oxidation time passes. Most widely-accepted interpretation of this phenomenon suggests that the decreasing amplitude of $1 \mathrm{NN} \mathrm{Pt-Pt}$ bond distance is caused by some of Pt-Pt bonds are elongating with few of them being contracted during Pt-O bonds formation. In similar way, we can also elaborate surface reconstruction or more precisely cluster reconstruction by analyzing $1 \mathrm{NN}$ distribution pattern using a simple model. The model was applied on the clusters by simply comparing the lengths of all bonds in the clusters before and after oxygen adsorption takes place as given in Figs. 4 and 5. The figures explicitly display the total sum of 1NN shell Pt-Pt bonds at certain bond length range. For Ni@Pt (pure Pt) cluster case, the two

green (red) high-intense peaks that assigned by A-B and B-B labels represent shell Pt-Pt bonds, while the background black line denotes the total sum of 1NN shell Pt-Pt bonds 
and the remaining interacting bonds in the clusters. The single black peak that located between them denotes the bonds that constructed by core and shell atoms $\left(a_{2}, a_{3}\right)$, and the remaining peaks on the left side of A-B peak are contribution from the core atoms $\left(\mathrm{a}_{0}, \mathrm{a}_{1}\right)$. Based on qualitative comparison, the double peaks of $1 \mathrm{NN}$ Pt-Pt bond distribution that plotted in Figures 4 and 5 may look contrast to single Gaussian-like peak at $2.77 \AA$ which routinely observed in FT graph of EXAFS measurement. This distinct discrepancy could stem from many factors such as geometric structure of $\mathrm{Pt}$ nanoparticles [25,53]. This can be explained as most of $1 \mathrm{NN}$ Pt-Pt bond distribution plots that reported in EXAFS measurement are not generated from single homogeneous structure of Pt nanoparticles but instead they are resulted from numerous non-uniform structures despite the average size of those $\mathrm{Pt}$ nanoparticles is relatively similar. However, the most reasonable reason that causes this striking discrepancy is size effect. Yevick and Frenkel [54] have demonstrated that the 1NN radial distribution function of Au cluster composed of 923 atoms ( $3.4 \mathrm{~nm}$ size) has continuous-like distribution pattern as opposed to the one consisting of 147 atoms ( $1.7 \mathrm{~nm}$ size) which acquires discrete-like distribution pattern. On the other hand, surface-induced strain effect that may exerted by $\mathrm{Al}_{2} \mathrm{O}_{3}$ surface on Pt-Pt bonds due to lattice mismatch imposes no significant influence on $1 \mathrm{~nm}$ Pt nanoparticles as reported by Nagai et al [55].

When oxygen adsorbed and induced moderate cluster reconstruction, the intensity of those A-B and B-B peaks are reduced since some of Pt-Pt bonds are slightly elongated and contracted by average value of $0.01 \AA$. This trivial bond length change produces new $1 \mathrm{NN}$ distribution patterns and these new patterns are also distinguishable from one to other adsorption sites on the same or different clusters. From overall observation, there is no shell Pt-Pt bonds elongation in Ni@Pt cluster that exceeds $3.1 \AA$. Similar result also obtained from pure Pt case except those from hcp-like and fcc-like sites where the low-intense peaks above the value $3.1 \AA$ are presumably coming from stretched (B-B)-assigned Pt-Pt bonds. Here, we assume that the coordination number $(\mathrm{CN})$ of Pt atoms remains unchanged unless stated otherwise, for instance, if the bonds are found to be broken. These results prove that the shell Pt-Pt bonds in $1 \mathrm{~nm} \mathrm{Ni@Pt}$ and pure Pt clusters are not as rigid as we have thought before despite the clusters can still robustly maintain their former structure.

\subsection{Cluster reconstruction in two-atom oxygen case}

In order to verify whether $1 \mathrm{NN}$ model is sufficient to discern the distribution patterns of 1NN Pt-Pt bonds between those induced by one or more oxygen atoms, we 
have further extended the investigation on two oxygen atoms case where the atoms are configured to resemble oxygen atoms that dissociated from molecular state. This atom rearrangement was intentionally reproduced to allow us to examine oxygen-induced reconstruction effect on both clusters that previously has been neglected in previous studies. As depicted in Fig. 6, four Pt-O bonds configurations are proposed and all of their structures have been fully relaxed.

Among those four configurations, C-01 of $\mathrm{Ni@Pt} \mathrm{cluster} \mathrm{clarified} \mathrm{our} \mathrm{early}$ prediction that vertex A site is harboring a metastable oxygen atom where the atom that initially resided on vertex A was displaced to hcp-like site after the structure was optimized. The displacement is more likely to occur as an effort to minimize repulsive lateral interaction between the neighboring oxygen atoms. On the other hand, the oxygen atoms in $\mathrm{C}-01$ of pure $\mathrm{Pt}$ cluster remain adhere to their initial positions, however unlike Ni@Pt case, the formation of Pt-O bonds has inflicting bond breaking process as indicated by three broken bonds (blue lines) in Fig. 6. For validation, calculations of C-01 of pure Pt cluster were carried out three times using different force criteria and all led to the same result. The standard DFT-based structure optimization is deemed to offer no guaranteed success in finding the stable structure. Such case should be more appropriate to be tackled by molecular dynamics treatment which has higher success rate, which unfortunately, such work is computationally time-consuming and does not serve the current objectives of this study. Hence, the current geometric structure of C-01 of pure Pt cluster is a metastable structure. Nevertheless, the result has demonstrated that core-shell Ni@Pt cluster is relatively more stable than pure Pt cluster. In addition, the oxygen adsorption energies of pure Pt clusters are also well comparable to the experimental work of Parker et al [56] (see Table 4) where at zero oxygen coverage the oxygen desorption energy of those dissociated oxygen atoms was found to be at 51 $\mathrm{kcal} / \mathrm{mol}$ or $2.21 \mathrm{eV}$. This good agreement between theoretical and experimental data fortifies the notion that dissociated oxygen atoms in small $\mathrm{Pt}$ nanoparticles are more preferably to choose edge-bridge sites over other sites.

Figure 7 shows 1NN Pt-Pt bond distributions in two-atom oxygen case. In comparison to one-atom oxygen case, it is obvious that none of these $1 \mathrm{NN}$ distribution patterns resemble those of one-atom oxygen case since the intensity of their A-B and B-B peaks is more deeply suppressed than the former case. The sole reason of differences between these two cases is caused by recursive effect of Pt-Pt bond length deviation as adding another oxygen atom onto the clusters surface will once more perpetuate cluster reconstruction. In particular case, the reconstruction could also lead to broken Pt-Pt bonds such the one found in C-01 of pure Pt cluster that followed by three 
prevalent yet unique traits on its $1 \mathrm{NN}$ Pt-Pt distribution pattern. First, the intensity of A-B and B-B peaks are strongly weakened as compared to the others. Second, there are more new emerging peaks at Pt-Pt bond distance range of 3.1 - $3.5 \AA$ while other configurations only have few of them. The last one is that beyond the limit value of 3.5 $\AA$ there are still other three peaks observed, one at $3.52 \AA$ (number 2 ) and the remaining two peaks at $3.59 \AA$ ( numbers 1 and 3). These three peaks are representing those three broken Pt-Pt bonds of $\mathrm{C}-01$ of pure Pt cluster that portrayed in Fig. 6. In addition, these peaks are located more closer to the vicinity of second nearest neighbor $(2 \mathrm{NN}) \mathrm{Pt}-\mathrm{Pt}$ bond distances of $3.92 \AA$ [57]. Hence, we argue that these three peaks are no longer belong to the group of $1 \mathrm{NN}$ Pt-Pt bond distribution and convincingly convey them as broken Pt-Pt bonds. Accordingly, the coordination number of those Pt atoms is also reduced.

While there is a contrast difference of $1 \mathrm{NN}$ Pt-Pt bond distribution pattern between each adsorption site in one-atom and two-atom adsorbed oxygen cases, the average Pt-O bond length that observed in both cases does not vary so much. The values are relatively close to the experimental value of $2 \AA$ as shown in Table 3 with exceptions those Pt-O bonds that established on vertex sites or C-01 of pure Pt cluster are slightly deviated. This proves that the length of Pt-O bonds is independent from the cluster size and alloying effects which agrees well with previous works [25,44,53]. Based on thorough analysis above, Pt-Pt bonds in pure Pt cluster are more prone to be distorted under the presence of oxygen rather than those contracted Pt-Pt bonds in core-shell Ni@Pt cluster, and this trait becomes more prevalent in two-atom oxygen case. Lastly, the general result shows that adsorbed oxygen is not only locally induced atom rearrangement near the surrounding adsorption site but also globally affected the connecting bonds of whole cluster atoms including the core atoms. This phenomenon is not limited to the oxidized Ni@Pt and pure Pt clusters only, similar observation had also been reported in oxidized $\mathrm{Na}_{55}$ cluster [58].

\subsection{Bader analysis of oxidized Ni@Pt and pure Pt clusters}

In this section, we discuss the bonding nature between the clusters and oxygen by using Bader charge analysis method. [59,60]. For one-atom oxygen case, unexpectedly, Bader charge analysis reveals that there is a general trend that indicates an increasing of charge transfer from $\mathrm{Pt}$ to $\mathrm{O}$ is proportionally equal to a steady increase of oxygen adsorption energy as observed in both clusters (see Table 5) with an exception in fcc-like site in Ni@Pt cluster where the trend is reversed. As additional 
information, the amount of charge transfer that received by oxygen on each adsorption site could also be used to identify the bonding character of Pt-O bonds formation. For instance, the charge transfer from $\mathrm{Pt}$ to $\mathrm{O}$ in vertex sites in pure Pt cluster is only about half of that in bulk PtO [51,61], indicating more covalent character instead of ionic character. However, the level of ionicity of Pt-O bonds approaches the bulk level as oxygen becomes strongly adsorbed on the clusters although the highest amount of charge transfer is still far below the value of bulk PtO. In overall, the ionicity level of $\mathrm{Pt}-\mathrm{O}$ bonds on pure Pt cluster varies and greatly depends on which adsorption site the oxygen is adsorbed. For the dissociated (two-atom) oxygen case, Pt-O bonds configuration on the clusters has strong similarity with Pt-O oxide structure of $\mathrm{Pt}_{\mathrm{x}} \mathrm{O}_{2 \mathrm{x}}$ cluster in term of the amount of total charge transfer. Based on the data provided in Table 6, the amount of charge transfer are twice of those in single atom cases. Another distinctive trait of $\mathrm{Pt}-\mathrm{O}$ bonds in dissociated oxygen atoms case is that the increment of charge transfer as the function of oxygen adsorption energy is nearly constant with the highest value is similar with the value $(-0.63 e)$ found in the smallest size of $\mathrm{Pt}_{\mathrm{x}} \mathrm{O}_{2 \mathrm{x}}$ cluster $\left(\mathrm{PtO}_{2}\right)$ [51]. Hence, it appears that most of Pt-O bonds are becoming more covalent with respect to those bonds in either bulk $\beta-\mathrm{PtO}_{2}$ or even bulk $\mathrm{PtO}$ when there is more than one adsorbed oxygen atom residing the clusters. This result may explain previous experimental work on $\mathrm{Pt} / \mathrm{Al}_{2} \mathrm{O}_{3}$ [62] where the surface oxide species that forms on $\mathrm{Pt}$ nanoparticles with diameters $<1.3 \mathrm{~nm}$ is $\mathrm{PtO}_{2}$. In addition, C-03 is the only configuration that does not follow the trend and further investigation is needed in order to address this matter.

In similar way, the explanation above can also be used to rationalize the same trend that observed on Ni@Pt cluster. Although no study has ever been conducted on bulk NiPt oxide due to such system might be unknown or non-existence, bulks PtO and $\beta-\mathrm{PtO}_{2}$ can still be used as reference to evaluate the ionicity level of Pt-O bonds on $\mathrm{Ni} @$ Pt cluster. The reason is that the oxide surface is still consisting of Pt-O bonds while there is no significant difference on the charge transfer between the one that transferred from Ni@Pt and pure Pt clusters. Therefore, the ionicity level of Pt-O bonds of oxidized Ni@Pt and pure Pt clusters is more or less the same regardless profound difference of their oxygen adsorption energy. Another consequence is that one would always be able to detect the presence of oxide forms such as $\mathrm{PtO}$ and $\mathrm{PtO}_{2}$ on the surface of $\mathrm{Ni} @ \mathrm{Pt}$ and pure Pt clusters where similar observation on other bimetallic Pt-M catalyst (where $\mathrm{M}=\mathrm{Au}, \mathrm{Pd}, \mathrm{Ru}, \mathrm{Fe}$ ) has been reported by XPS spectra results. [63] 


\section{Conclusions}

In summary, we have examined the core-shell structure of $\mathrm{Ni@Pt}$ and its corresponding pure Pt cluster to investigate the preferable adsorption sites for oxygen atoms. In general, the oxygen adsorption energy is mainly governed by (1) atomic configuration of Pt-O bonds, (2) alloying and (3) cluster reconstruction effects. Based on oxygen adsorption energy and $v(\mathrm{Pt}-\mathrm{O})$ stretching frequency calculations and comparison with available experimental data, the global trend of Pt-O bonding strength in the clusters is more likely to follow successive order of bridge (and/or hcp-like) > fcc-like $>$ vertex A > vertex B where the vertex sites are presumed to harbor "metastable" oxygen atoms. In overall, core-shell Ni@Pt cluster is able to reduce Pt-O bonding strength despite on bridge site case the adsorption energy is relatively similar to the one in pure Pt cluster.

We also have shown that the 1NN model can qualitatively be used to compare the changes in 1NN Pt-Pt bond distribution patterns with the one of Fourier transformed of EXAFS data. Although there are some noticeable differences between these two types of data because of several factors, the general trend of our results and those of experimental works are vividly alike where the amplitude of $1 \mathrm{NN}$ Pt-Pt bond distribution of surface atoms would decrease as oxygen absorbed on the clusters. Moreover, oxygen-induced cluster reconstruction affects both shell Pt-Pt bond lengths near the proximity of the adsorption site and globally the lengths of all interacting bonds that are located at great distance from the position of Pt-O bonds formation. In addition, the average length of $\mathrm{Pt}-\mathrm{O}$ bonds in the cluster is similar with the experimental values. In term of structural robustness, the role of $\mathrm{Ni}$ core atoms in core-shell $\mathrm{Ni}$ @ Pt is to prevent bond breaking process on shell Pt-Pt bonds. Finally, based on Bader charge analysis, the majority bonding character of Pt-O bonds in the clusters is covalent. However, more ionic characters might occur as Pt-O bonding strengths are increased.

\section{Acknowledgements}

This work is supported in part by MEXT Grant-in-Aid for Scientific Research on Innovative Areas Program (2203-22104008) and Scientific Research programs (A) (24246013); JST ALCA Program "Development of Novel Metal-Air Secondary Battery Based on Fast Oxide Ion Conductor Nano Thickness Film", Strategic Japan-Croatia Research Cooperative Program on Materials Sciences Program "Theoretical Modeling and Simulations of the Structural, Electronic, and Dynamical Properties of Surfaces and 
Nanostructures in Materials Science Research, and ISUZU. Some of the numerical calculations presented here done using the computer facilities at the following institutes: CMC (Osaka University), ISSP, KEK, NIFS, and YITP.

\section{References}

[1] M. Moldovan, M. A. Palacios, M. M. Gómez, G. Morrison, S. Rauch, C. McLeod, R. Ma, S. Caroli, A. Alimonti, F. Petrucci, B. Bocca, P. Schramel, M. Zischka, C. Pettersson, U. Wass, M. Luna, J. C. Saenz, J. Santamaría, Sci. Total Environ. 296 (2002) 199-208.

[2] S. Mulla, N. Chen, L. Cumaranatunge, G. Blau, D. Zemlyanov, W. Delgass, W. Epling, F. Ribeiro, J. Catal. 241 (2006) 389-399.

[3] C. Mager-Maury, G. Bonnard, C. Chizallet, P. Sautet, P. Raybaud, ChemCatChem 3 (2011) 200-207.

[4] F. C. Galisteo, R. Mariscal, M. L. Granados, M. D. Z. poves, J. L. G. Fierro, V. Kroger, R. L. Keiski, Appl. Catal. B 72 (2007) 272-281.

[5] H. Shinjoh, Catal. Surv. from Asia 13 (2009) 184-190.

[6] S. R. de Miguel, I. M. J. Vilella, S. P. Maina, D. San José-Alonso, M. C. Román-Martínez, M. J. Illán-Gómez, Appl. Catal. A 435-436 (2012) 10-18.

[7] M. García-Diéguez, E. Finocchio, M. Á. Larrubia, L. J. Alemany, G. Busca, J. Catal. 274 (2010) 11-20.

[8] E. Örücü, F. Gökaliler, A. E. Aksoylu, Z. I. Önsan, Catal. Letters 20 (2007) 198203.

[9] B. S. Çağlayan, Z. İ. Önsan, A. E. Aksoylu, Catal. Letters 102 (2006) 63-67.

[10] J. Li, W. P. Tian, X. Wang, L. Shi, Chem. Eng. J. 175 (2011) 417-422.

[11] L. Olsson, H. Persson, E. Fridell, M. Skoglundh, B. Andersson, J. Phys. Chem. B 105 (2001) 6895-6906. 
[12] M. Crocoll, S. Kureti, W. Weisweiler, J. Catal. 229 (2005) 480-489.

[13] F. Mittendorfer, A. Eichler, J. Hafner, Surf. Sci. 433-435 (1999) 756-760.

[14] G. Wang, H. Wu, D. Wexler, H. Liu, O. Savadogo, J. Alloys Compd. 503 (2010) L1-L4.

[15] Y. Chen, F. Yang, Y. Dai, W. Wang, S. Chen, J. Phys. Chem. C 112 (2008) 16451649.

[16] J. Snyder, T. Fujita, M. W. Chen, J. Erlebacher, Nat. Mater. 9 (2010) 904-907.

[17] A. Boubnov, S. Dahl, E. Johnson, A. P. Molina, S. B. Simonsen, F. M. Cano, S. Helveg, L. J. Lemus-Yegres, J.-D. Grunwaldt, Appl. Catal. B 126 (2012) 315325 .

[18] B. M. Weiss, E. Iglesia, J. Phys. Chem. C 113 (2009) 13331-13340.

[19] B. Han, C. Miranda, G. Ceder, Phys. Rev. B 77 (2008) 075410.

[20] J. Kitchin, J. Nørskov, M. Barteau, J. Chen, Phys. Rev. Lett. 93 (2004) 4-7.

[21] P. G. Allen, S. D. Conradson, M. S. Wilson, S. Gottesfel, I. D. Raistrick, J. Valerio, M. Lovato, J. Electroanal. Chem. 384 (1995) 99-103.

[22] H. Imai, K. Izumi, M. Matsumoto, Y. Kubo, K. Kato, Y. Imai, J. Am. Chem. Soc. 131 (2009) 6293-6300.

[23] O. S. Alexeev, S. Y. Chin, M. H. Engelhard, L. Ortiz-Soto, M. D. Amiridis, J. Phys. Chem. B 109 (2005) 23430-23443.

[24] J. H. Kang, L. D. Menard, R. G. Nuzzo, A. I. Frenkel, J. Am. Chem. Soc. 128 (2006) 12068-12069.

[25] S. Mostafa, F. Behafarid, J. R. Croy, L. K. Ono, L. Li, J. C. Yang, A. I. Frenkel, B. R. Cuenya, J. Am. Chem. Soc. 132 (2010) 15714-15719.

[26] S. Chen, J. P. K. Doyea, D. J. Wales, New J. Chem. (1998) 733-744. 
[27] L.-L. Wang, D. D. Johnson, J. Am. Chem. Soc. 131 (2009) 14023-14029.

[28] J. Wu, L. Qi, H. You, A. Gross, J. Li, H. Yang, J. Am. Chem. Soc. 134 (2012) $11880-11883$.

[29] G. Kresse, J. Hafner, Phys. Rev. B 47 (1993) 558-561.

[30] G. Kresse, J. Hafner, Phys. Rev. B 48 (1993) 13115-13118.

[31] G. Kresse, J. Hafner, Phys. Rev. B 49 (1994) 251-269.

[32] J. Perdew, K. Burke, M. Ernzerhof, Phys. Rev. Lett. 77 (1996) 3865-3868.

[33] D. Karhánek, T. Bučko, J. Hafner, J. Phys. Condens. Matter 22 (2010) 265006.

[34] S. K. Nayak, S. N. Khanna, B. K. Rao, P. Jena, J. Phys. Chem. A 101 (1997) 1072-1080.

[35] L. Xiao and L. Wang, J. Phys. Chem. A 108 (2004) 8605-8614.

[36] S. Mukerjee, S. Srinivasan, M. P. Soriaga, J. McBreen, J. Phys. Chem. 99 (1995) 4577-4589.

[37] M. Teliska, V. S. Murthi, S. Mukerjee, D. E. Ramaker, J. Electrochem. Soc. 152 (2005) A2159-A2169.

[38] J. L. Gland, B. A. Sexton, G. B. Fisher, Surf. Sci. 95 (1980) 587-602.

[39] P. Gambardella, Ž. Šljivančanin, B. Hammer, M. Blanc, K. Kuhnke, K. Kern, Phys. Rev. Lett. 87 (2001) 1-4.

[40] P. Feibelman, Phys. Rev. B 56 (1997) 10532-10537.

[41] Z. Gu, P. B. Balbuena, J. Phys. Chem. C 111 (2007) 9877-9883.

[42] J. M. Bray, W. F. Schneider, Langmuir 27 (2011) 8177-8186.

[43] M. R. McClellan, F. R. Mcfeely, Surf. Sci. 123 (1983) 188-208.

[44] T. Jacob, B. V. Merinov, W. A. Goddard, Chem. Phys. Lett. 385 (2004) 374-377. 
[45] H. Wang, R. G. Tobin, D. K. Lambert, C. L. DiMaggio, G. B. Fisher, Surf. Sci. 372 (1997) 267-278.

[46] F. Oemry, A. A. B. Padama, H. Kishi, S. Kunikata, H. Nakanishi, H. Kasai, H. Maekawa, K. Osumi, K. Sato, Jpn. J. Appl. Phys. 51 (2012) 035002.

[47] A. Eichler, J. Hafner, Phys. Rev. Lett. 79 (1997) 4481-4484.

[48] G. A. Somorjai, Langmuir 7 (1991) 3176-3182.

[49] G. A. Somorjai, Surf. Sci. 242 (1991) 481-488.

[50] M. A. Van Hove, G. A. Somorjai, Surf. Sci. 299/300 (1994) 487-501.

[51] Y. Xu, W. A. Shelton, W. F. Schneider, J. Phys. Chem. A 110 (2006) 58395846.

[52] P. Rzeszotarski, Z. Kaszkur, Phys. Chem. Chem. Phys. 11 (2009) 5416-5421.

[53] B. R. Cuenya, J. R. Croy, S. Mostafa, F. Behafarid, L. Li, Z. Zhang, J. C. Yang, Q. Wang, A. I. Frenkel, J. Am. Chem. Soc. 132 (2010) 8747-8756.

[54] A. Yevick, A. I. Frenkel, Phys. Rev. B 81 (2010) 115451.

[55] Y. Nagai, T. Hirabayashi, K. Dohmae, N. Takagi, T. Minami, H. Shinjoh, S. Matsumoto, J. Catal. 242 (2006) 103-109.

[56] D. H. Parker, M. E. Bartram, B. E. Koel, Surf. Sci. 217 (1989) 489-510.

[57] A. Jentys, Phys. Chem. Chem. Phys. 1 (1999) 4059-4063.

[58] C. Hock, S. Straßburg, H. Haberland, B. V. Issendorff, A. Aguado, M. Schmidt, Phys. Rev. Lett. 101 (2008) 023401.

[59] G. Henkelman, A. Arnaldsson, H. Jónsson, Comput. Mater. Sci. 36 (2006) 354 360.

[60] R. F. W. Bader, Atoms in Molecules: A Quantum Theory, Oxford University Press, Oxford, 1990. 
[61] N. Seriani, Z. Jin, W. Pompe, L. Ciacchi, Phys. Rev. B 76 (2007) 155421.

[62] C.-B. Wang, C.-T. Yeh, J. Catal. 178 (1998) 450-456.

[63] J. R. Croy, S. Mostafa, L. Hickman, H. Heinrich, B. R. Cuenya, Appl. Catal. A 350 (2008) 207-216. 


\section{Figures Caption}

Fig. 1. Optimized structures of core-shell Ni@Pt (a) and pure Pt (b) clusters with embedded red and black lines represent two varying Pt-Pt bond distances. The schematic cross-section of the clusters structure (c) exhibits the connecting bonds between atoms vary in length as indicated by characters $a_{n}(n=0,1,2,3,4)$ where their values are provided in Table 1 . The 13-atom clusters on the left and right sides serve as the core atoms of Ni@Pt and pure Pt clusters. Platinum and nickel atoms are represented by gray and green spheres.

Fig. 2. Small representative structures of core-shell Ni@Pt (left) and pure Pt (right) clusters that are taken from the clusters in Figs. 1 (a) and 1 (b). The numbers denote oxygen adsorption sites.

Fig. 3. Oxygen adsorption energies on various designated adsorption sites as shown in Fig. 2. Green (black) dashed and solid lines correspond to core-shell Ni@Pt (pure Pt) cluster for rigid and reconstructed cluster cases, respectively. See the text for more details.

Fig. 4. First nearest neighbor (1NN) Pt-Pt bond distribution in core-shell Ni@Pt cluster for oneatom oxygen case. Black and green lines correspond to total atom-atom bonds in the cluster and shell Pt-Pt bonds, respectively.

Fig. 5. First nearest neighbor $(1 \mathrm{NN}) \mathrm{Pt}-\mathrm{Pt}$ bond distribution in pure Pt cluster for one-atom oxygen case. Black and red lines correspond to total atom-atom bonds in the cluster and shell PtPt bonds, respectively

Fig. 6. Geometric configurations of dissociated (two-atom) oxygen in reconstructed core-shell $\mathrm{Ni@Pt} \mathrm{and} \mathrm{pure} \mathrm{Pt} \mathrm{clusters.}$

Fig. 7. First nearest neighbor (1NN) Pt-Pt bond distribution in core-shell Ni@Pt (top panels) and pure Pt clusters (bottom panels) for two-atom oxygen case. For details, please refer to Figs. 4 and 5. 
Figure 1
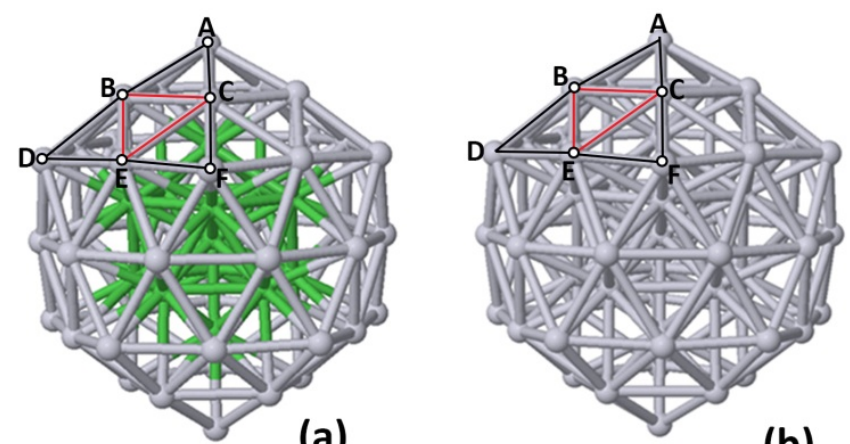

(a)

(b)

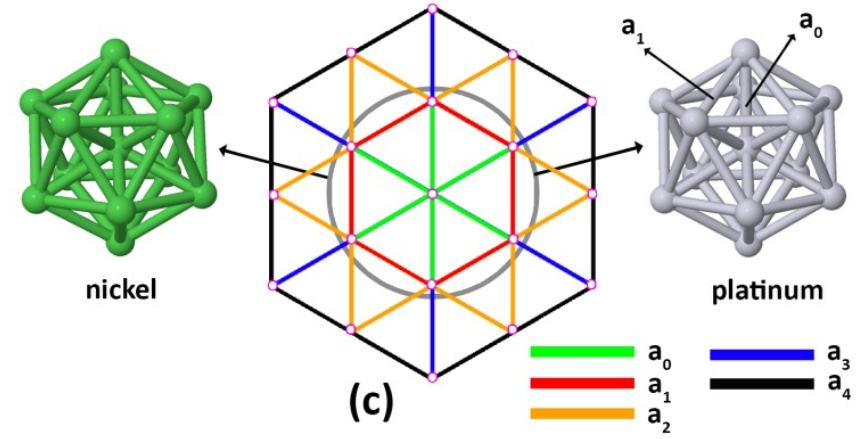

Figure 2

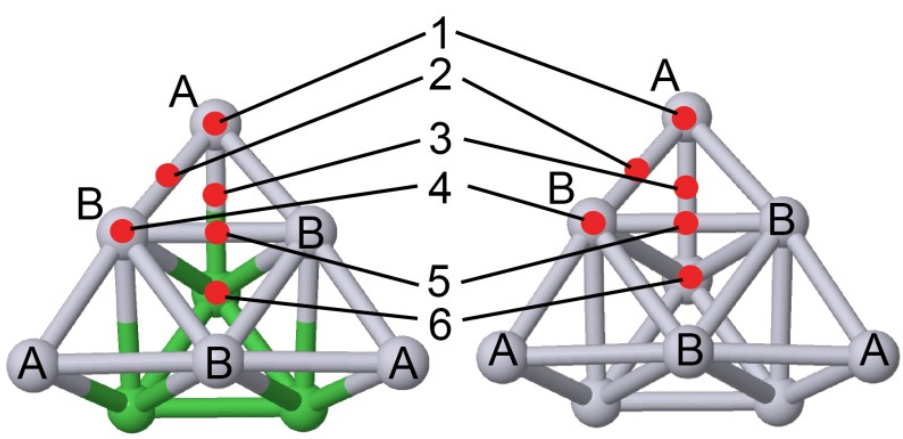


Figure 3

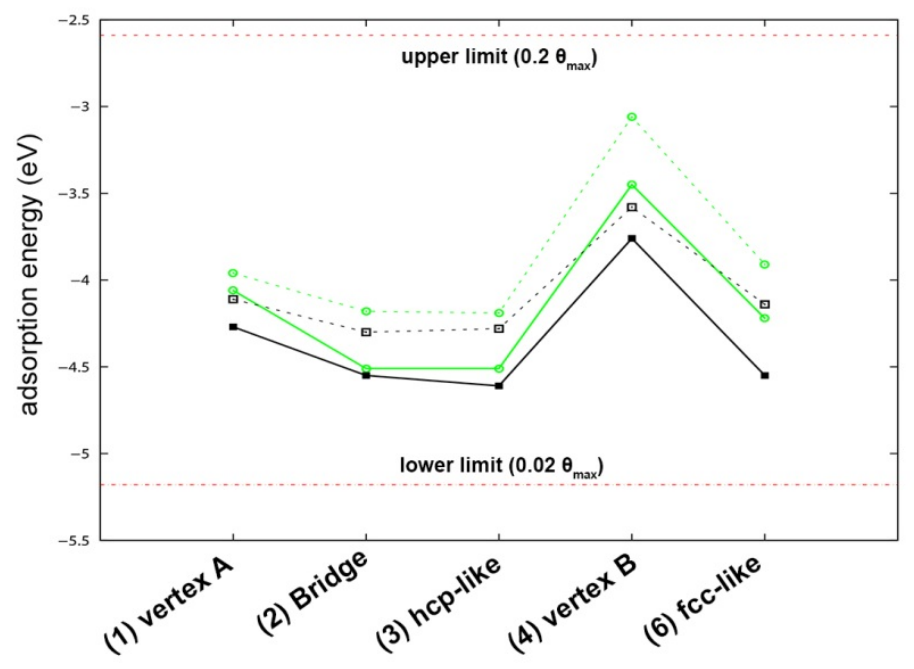

Figure 4

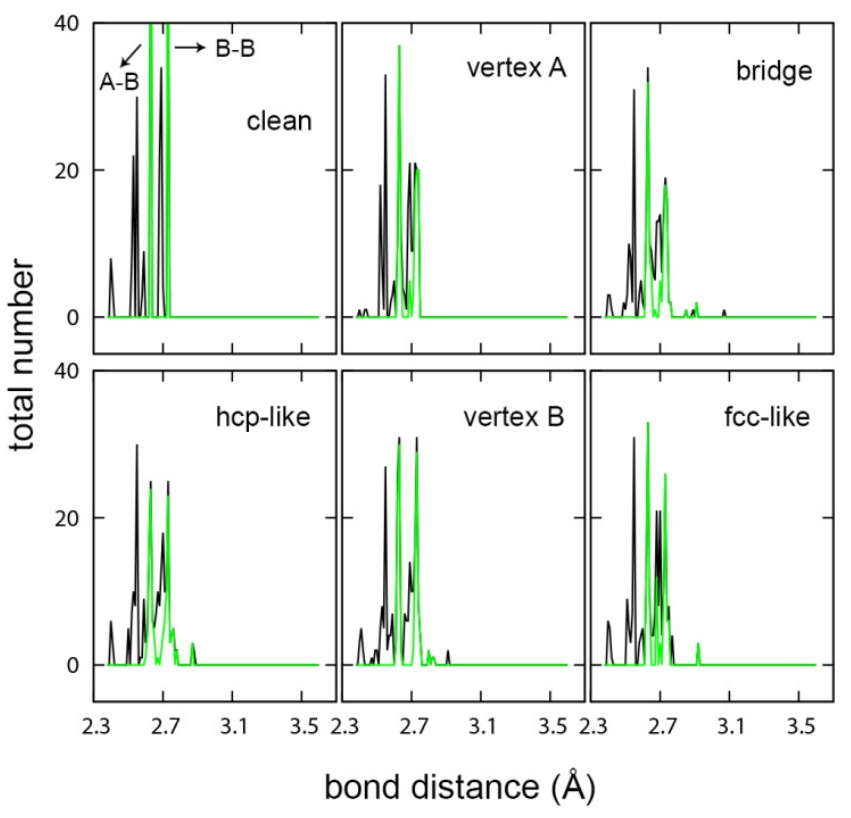


Figure 5

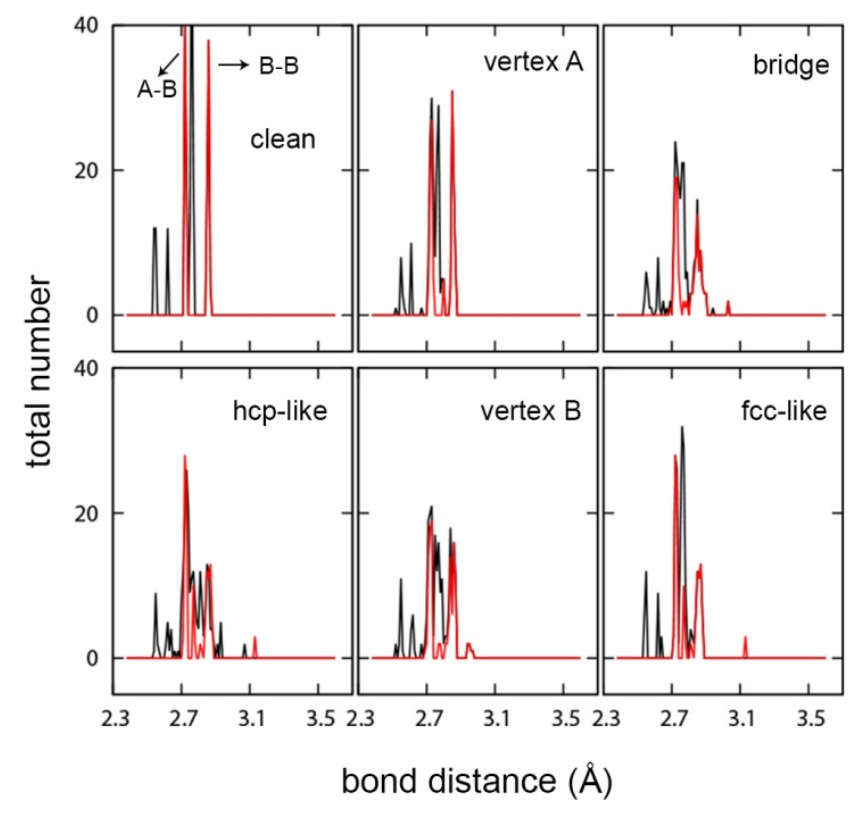

Figure 6
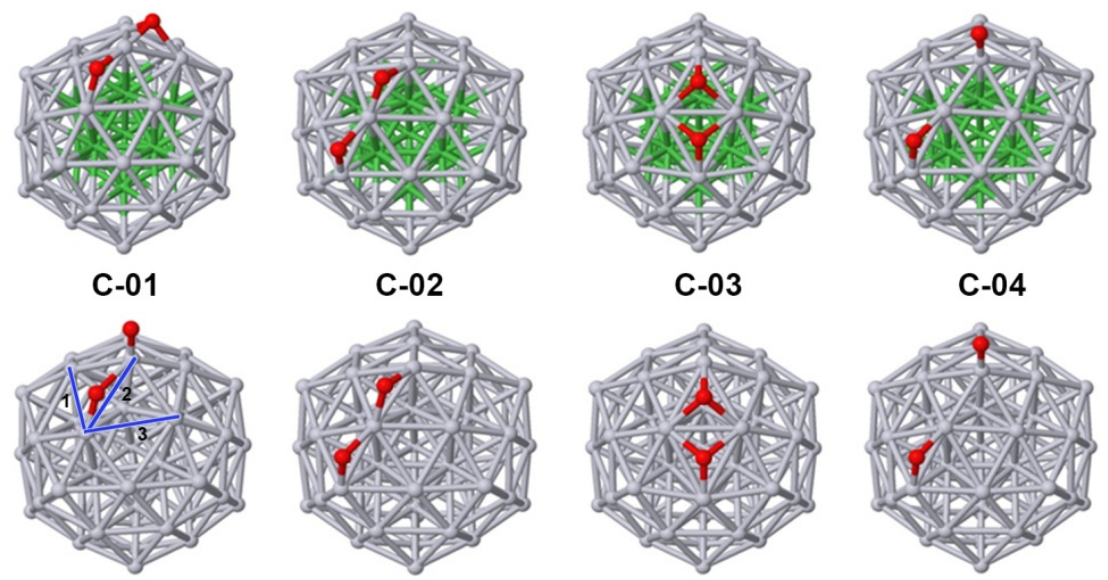
Figure 7

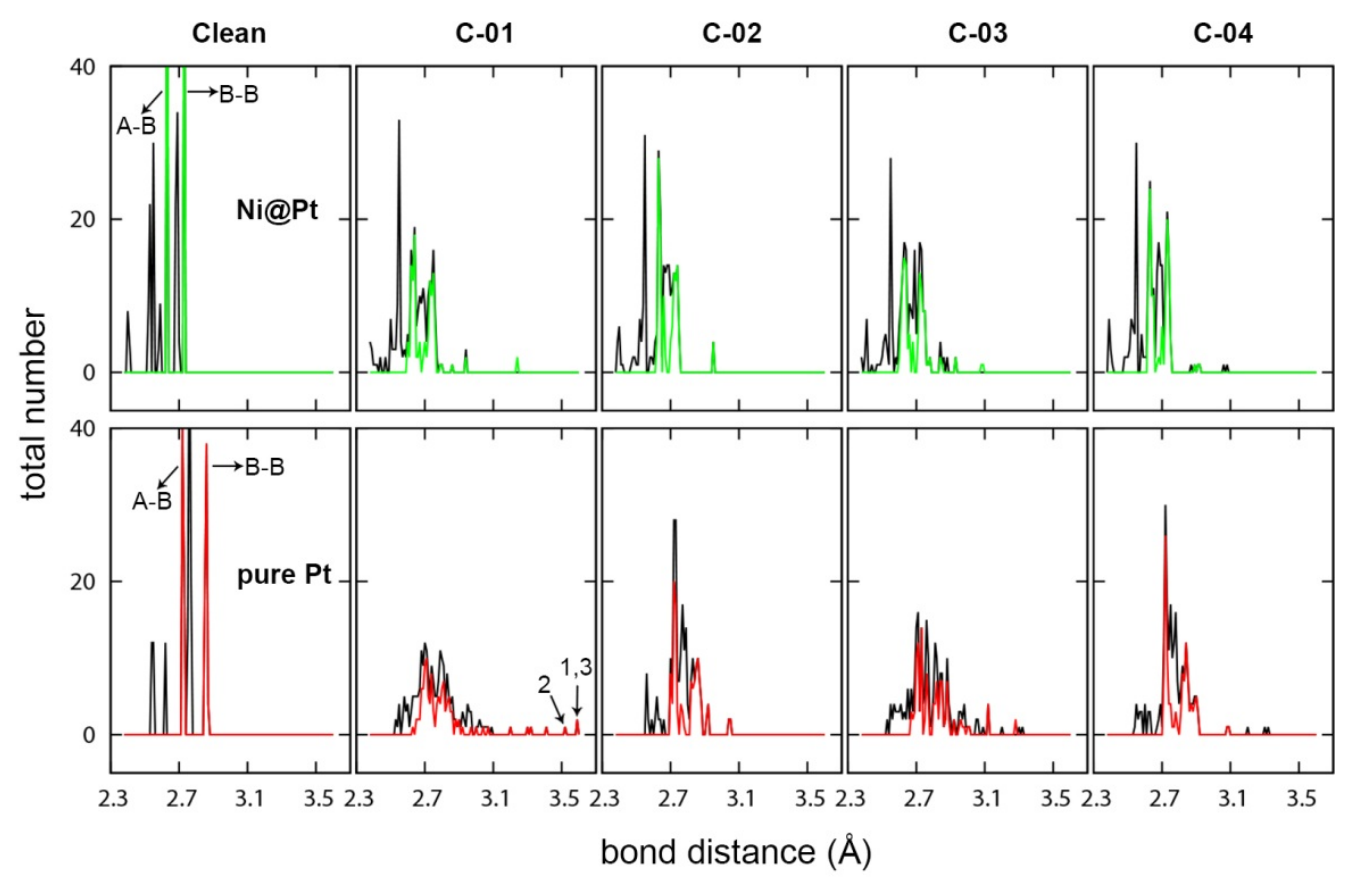




\section{Table 1}

Atom-atom bond distances of the bonds in core-shell Ni@Pt and pure Pt clusters that shown in Figure $1(\mathrm{c})$ and denoted by characters $\mathrm{a}_{\mathrm{n}}(\mathrm{n}=0,1,2,3,4)$. The unit is given in $\AA$.

\begin{tabular}{ccc}
\hline & $\mathrm{Ni} @$ Pt & Pure Pt \\
\hline $\mathrm{a}_{0}$ & 2.40 & 2.62 \\
& $2.32^{*}$ & $2.63^{*}$ \\
$\mathrm{a}_{1}$ & 2.53 & 2.76 \\
& $2.44^{*}$ & $2.77^{*}$ \\
$\mathrm{a}_{2}$ & 2.69 & 2.76 \\
$\mathrm{a}_{3}$ & 2.59 & 2.54 \\
$\mathrm{a}_{4}$ & 2.68 & 2.79 \\
$\mathrm{a}_{4}$ (exp) [36,37] & $2.70(5 \mathrm{~nm})$ & $2.77(3 \mathrm{~nm})$ \\
\hline *refers to stable 13-atom cluster &
\end{tabular}

Table 2

Vibrational Pt-O stretching frequency for adsorbed oxygen atom in reconstructed $\mathrm{Ni@Pt} \mathrm{and}$ pure Pt clusters

\begin{tabular}{|c|c|c|c|c|}
\hline cluster/surface & $\begin{array}{c}\text { Site } \\
\text { position }\end{array}$ & $\begin{array}{l}\text { Adsorption } \\
\text { energy } \\
(\mathrm{eV})\end{array}$ & $\begin{array}{l}\text { frequency } \\
\left(\mathrm{cm}^{-1}\right)\end{array}$ & $\begin{array}{c}\text { Frequency } \\
\text { gap } \\
\left(\mathrm{cm}^{-1}\right)\end{array}$ \\
\hline \multirow[t]{5}{*}{ Ni@Pt } & vertex B & -3.45 & 722 & 54 \\
\hline & vertex A & -4.06 & 787 & \\
\hline & fcc-like & -4.22 & 451 & \\
\hline & bridge & -4.51 & $492^{a}$ & \\
\hline & hcp-like & -4.51 & $546^{\mathrm{a}}$ & \\
\hline \multirow[t]{5}{*}{ Pure Pt } & vertex B & -3.76 & 725 & 73 \\
\hline & vertex A & -4.27 & 752 & \\
\hline & fcc-like & -4.55 & 405 & \\
\hline & bridge & -4.51 & $447^{\mathbf{b}}$ & \\
\hline & hcp-like & -4.61 & $520^{b}$ & \\
\hline $\operatorname{Pt}(335)(\exp )$ & terrace & - & $470^{c}$ & 90 \\
\hline \multirow[t]{2}{*}[45]{} & step & - & $560^{\mathrm{c}}$ & \\
\hline & step & - & 700 & \\
\hline $\mathrm{Pt}_{4}[46]$ & bridge & - & 666 (TS) & \\
\hline $\mathrm{Pt}_{10}[46]$ & bridge & - & 693 (TS) & \\
\hline
\end{tabular}

Note: the frequency gap is calculated by taking frequency difference between the frequencies that assigned by superscript $\mathbf{a}, \mathbf{b}$, and $\mathbf{c}$. TS is abbreviation for "transition state". 
Table 3

Average Pt-O bond length energies in reconstructed core-shell Ni@Pt and pure Pt clusters. The unit is given in $\AA$.

\begin{tabular}{ccc}
\hline & Ni@Pt & Pure Pt \\
\hline Vertex A & 1.77 & 1.80 \\
Bridge & 1.95 & 1.96 \\
hcp-like & 2.05 & 2.06 \\
Vertex B & 1.81 & 1.82 \\
fcc-like & 2.07 & 2.07 \\
C-01 & 2.03 & $1.79^{\mathbf{a}}$ \\
& 1.92 & $1.85^{\mathbf{a}}$ \\
C-02 & 1.95 & 1.96 \\
& 1.95 & 1.96 \\
C-03 & 2.09 & 2.08 \\
& 2.08 & 2.09 \\
C-04 & $1.78^{\mathbf{b}}$ & $1.79^{\mathbf{b}}$ \\
& 1.95 & 1.96 \\
EXAFS [22] & N/A & 2.00 \\
\hline oxygen atom that adsorbed on metastable structure ${ }^{\mathrm{a}}$ and vertex A site
\end{tabular}

Table 4

Oxygen adsorption energies in $\mathrm{Ni} @ \mathrm{Pt}$ and pure Pt clusters for two-atom oxygen case

\begin{tabular}{lllll} 
& $\mathrm{C}-01$ & $\mathrm{C}-02$ & $\mathrm{C}-03$ & $\mathrm{C}-04$ \\
\cline { 2 - 5 } $\mathrm{Ni} @ \mathrm{Pt}$ & -2.45 & -2.20 & -1.30 & -1.80 \\
Pure Pt & $\mathrm{N} / \mathrm{A}^{*}$ & -2.29 & -1.90 & -2.18 \\
\hline
\end{tabular}

Not avalaible 


\section{Table 5}

Bader atomic charges for one-atom oxygen case

\begin{tabular}{ccccc}
\hline cluster & $\begin{array}{c}\text { adsorption } \\
\text { site }\end{array}$ & $\begin{array}{c}\text { charge }(e) \\
\text { on cluster }\end{array}$ & $\begin{array}{c}\text { charge }(e) \\
\text { on O atom }\end{array}$ & $\begin{array}{c}\text { adsorption } \\
\text { energy }(\mathrm{eV})\end{array}$ \\
\hline $\mathrm{Ni} @ \mathrm{Pt}$ & vertex B & +0.45 & -0.45 & -3.45 \\
& vertex A & +0.58 & -0.58 & -4.06 \\
& bridge & +0.63 & -0.63 & -4.51 \\
& fcc-like & +0.71 & -0.71 & -4.22 \\
& hcp-like & +0.76 & -0.76 & -4.51 \\
pure Pt & vertex B & +0.48 & -0.48 & -3.76 \\
& vertex A & +0.47 & -0.47 & -4.27 \\
& bridge & +0.63 & -0.63 & -4.55 \\
& fcc-like & +0.68 & -0.68 & -4.55 \\
hcp-like & +0.73 & -0.73 & -4.61 \\
PtO & & & & \\
Bulk PtO & & $+0.48^{\mathrm{a}}$ & $-0.48^{\mathrm{a}}$ & \\
& & $+0.96^{\mathrm{a}}$ & $-0.96^{\mathrm{a}}$ & \\
& & $+0.86^{\mathrm{b}}$ & $-0.86^{\mathrm{b}}$ & \\
\hline
\end{tabular}

See references $[51]^{\mathrm{a}}$ and $[61]^{\mathrm{b}}$

\section{Table 6}

Bader atomic charges for two-atom oxygen case

\begin{tabular}{ccccc}
\hline cluster & $\begin{array}{c}\text { adsorption } \\
\text { site }\end{array}$ & $\begin{array}{c}\text { charge }(e) \\
\text { on cluster }\end{array}$ & $\begin{array}{c}\text { charge }(e)^{*} \\
\text { on O atom }\end{array}$ & $\begin{array}{c}\text { adsorption } \\
\text { energy }(\mathrm{eV})\end{array}$ \\
\hline $\mathrm{Ni} @ \mathrm{Pt}$ & $\mathrm{C}-04$ & +1.18 & -0.59 & -1.8 \\
& $\mathrm{C}-02$ & +1.24 & -0.62 & -2.2 \\
& $\mathrm{C}-03$ & +1.3 & -0.65 & -1.3 \\
& $\mathrm{C}-01$ & +1.37 & -0.69 & -2.45 \\
pure Pt & $\mathrm{C}-04$ & +1.2 & -0.6 & -2.18 \\
& $\mathrm{C}-02$ & +1.24 & -0.62 & -2.29 \\
& $\mathrm{C}-03$ & +1.32 & -0.66 & -1.9 \\
$\mathrm{PtO}_{2}$ & & & & \\
$\mathrm{Bulk} \beta-\mathrm{PtO}_{2}$ & & $+1.27^{\mathrm{a}}$ & $-0.63^{\mathrm{a}}$ & \\
& & $+1.70^{\mathrm{a}}$ & $-0.85^{\mathrm{a}}$ & \\
& & $+1.62^{\mathrm{b}}$ & $-0.81^{\mathrm{b}}$ & \\
\hline
\end{tabular}

${ }^{*}$ For the sake of clarity, the average charge of two $\mathrm{O}$ atoms is reported. C-01 in pure Pt is excluded from the list since its oxygen adsorption energy could not be obtained. See references [51] and [61] $]^{\mathrm{b}}$ 

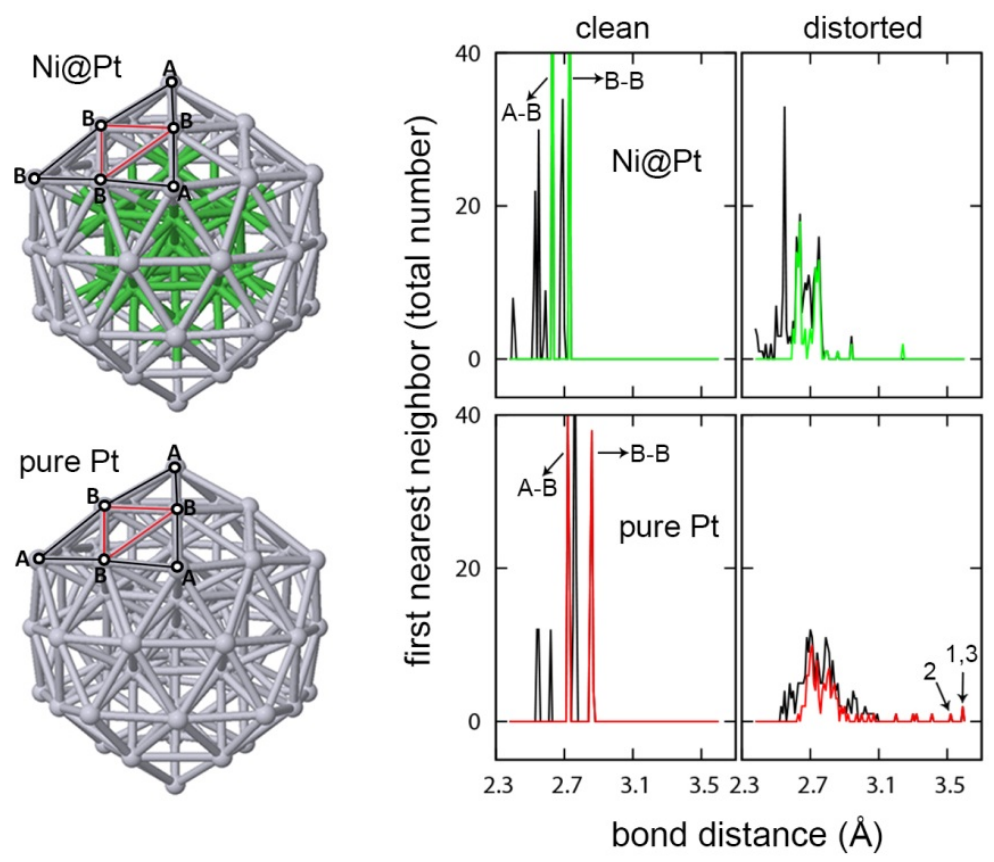